JoAnna RenCeAWOWicz and Wojciech M. ZająCZKOWski (Warszawa)

\title{
EXISTENCE OF SOLUTIONS TO THE POISSON EQUATION IN $L_{2}$-WEIGHTED SPACES
}

Abstract. We consider the Poisson equation with the Dirichlet and the Neumann boundary conditions in weighted Sobolev spaces. The weight is a positive power of the distance to a distinguished plane. We prove the existence of solutions in a suitably defined weighted space.

1. Introduction. We study the following boundary value problem for the Poisson equation in weighted spaces:

$$
\begin{aligned}
& -\Delta u=f \quad \text { in } \Omega, \\
& u_{,\left.x_{3}\right|_{S_{*}}}=0, \\
& \left.u\right|_{S_{1}}=0, \\
& \left.u\right|_{S_{0}}=0,
\end{aligned}
$$

where $\Omega \subset \mathbb{R}^{3}, \partial \Omega=S_{0} \cup S_{1} \cup S_{*}=S$ (see Fig. 1).

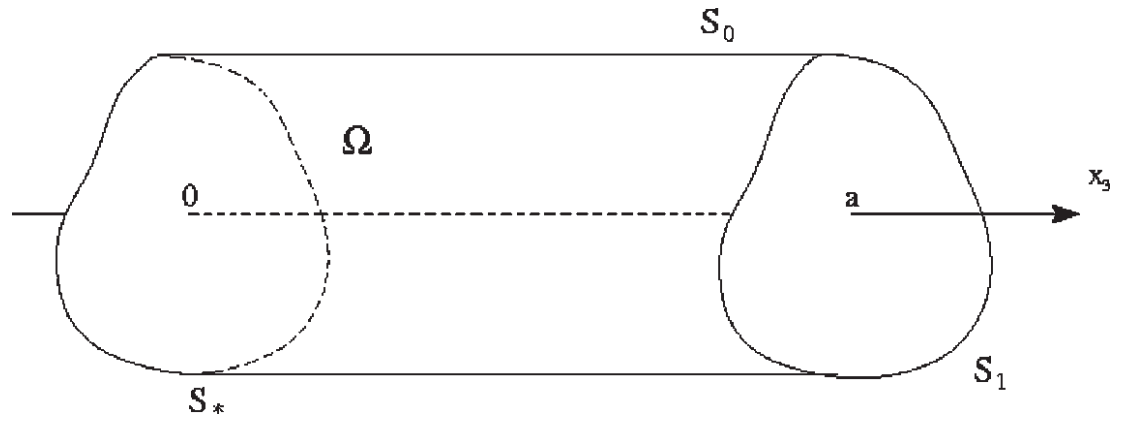

Fig. 1. Domain $\Omega$

2010 Mathematics Subject Classification: Primary 35J05; Secondary 31A30, 35J25.

Key words and phrases: Poisson equation, weighted Sobolev spaces, Neumann boundaryvalue problem, Dirichlet boundary-value problem. 
Here, $S_{0}$ is parallel and $S_{1}$ and $S_{*}$ are perpendicular to the $x_{3}$ axis, and $S_{1}$ meets the $x_{3}$ axis at the point $x_{3}=a, a<\infty$, while $S_{*}$ meets the $x_{3}$ axis at the point $x_{3}=0$.

The problem arises as an auxiliary system in the analysis of the inflowoutflow motion described by the Navier-Stokes equations and the aim of this paper is to prepare tools to examine the NS equations. We want to use the estimates derived here to remove some restrictions on the boundary inflow for the Navier-Stokes system.

Let us consider the Navier-Stokes equations with slip boundary conditions on $S=S_{0} \cup S_{1} \cup S_{*}$ with inflow on $S_{*}$ and outflow on $S_{1}$. On $S_{0}$ the normal component of the velocity vanishes. The inflow-outflow problem is a difficult and unclear problem for NS (see [L, Ch. 5] and [G, Vol. 2, Ch. 8]. Usually, such a result requires strong restrictions, for example in the proof of global existence in [Z2] the inflow flux either must vanish or must be sufficiently small in time. Our goal is to prove long time existence of solutions to the Navier-Stokes equations without restrictions on the magnitude of the inflow flux. The first and most crucial step is to obtain a global estimate (with nonvanishing inflow flux) for weak solutions. To this end, we homogenize the problem in question by solutions of some elliptic systems where the function $\eta$ (see [L, Ch. 5, Sect. 4]) is used. The derivative of this function implies the weight introduced in this paper. Moreover, to estimate the nonlinear terms which correspond to $v \cdot \nabla v$, where $v$ is the velocity of the fluid, we need an $L_{p}$ version, with $p=3$, of Theorem 1 (see [RZ]).

To formulate the main result of this paper we introduce

$$
H_{\mu}^{k}(\Omega)=\left\{u:\|u\|_{H_{\mu}^{k}(\Omega)}=\left(\sum_{|\alpha| \leq k} \int_{\Omega} d x^{\prime} d x_{3}\left|D_{x}^{\alpha} u\right|^{2} x_{3}^{2(\mu+|\alpha|-k)}\right)^{1 / 2}<\infty\right\},
$$

where $k \in \mathbb{N}_{0}=\mathbb{N} \cup\{0\}, \mu \in \mathbb{R}, \alpha=\left(\alpha_{1}, \alpha_{2}, \alpha_{3}\right)$ is a multiindex, $|\alpha|=$ $\alpha_{1}+\alpha_{2}+\alpha_{3}, \alpha_{i} \in \mathbb{N}_{0}, i=1,2,3, D_{x}^{\alpha}=\partial_{x_{1}}^{\alpha_{1}} \partial_{x_{2}}^{\alpha_{2}} \partial_{x_{3}}^{\alpha_{3}}$. Moreover, we denote $L_{2, \mu}(\Omega)=H_{\mu}^{0}(\Omega)$.

Note, that $x_{3}^{p \mu}, p \in(1, \infty)$, is not a Muckenhoupt weight so the results of Coifman-Fefferman $[\mathrm{CF}$ cannot be applied.

Theorem 1. Assume that $f \in L_{2, \mu}(\Omega)$ can be expressed in the form

$$
f=\alpha_{, x_{3}} \quad \text { with } \quad \alpha=\tilde{a} \eta\left(x_{3}\right),\left.\tilde{a}\right|_{x_{3}=0}=\theta,
$$

where

(i) $\eta\left(x_{3}\right)$ is a smooth non-increasing function with compact support such that $0 \leq \eta \leq 1$ and $\eta=1$ in a neighbourhood of $x_{3}=0$,

(ii) $\theta \in H^{1}\left(S_{*}\right)$,

(iii) $\tilde{a}$ is an extension of $\theta$ to $x_{3} \geq 0$ such that

$$
\|\tilde{a}\|_{L_{\infty}\left(\mathbb{R}_{+} ; H^{1}\left(S_{*}\right)\right)} \leq c\|\theta\|_{H^{1}\left(S_{*}\right)} .
$$


Then a solution $u$ of the problem (1.1) exists and satisfies

$$
\|u-u(0)\|_{H_{\mu}^{2}(\Omega)} \leq c\left(\|f\|_{L_{2, \mu}(\Omega)}+\|\theta\|_{H^{1}\left(S_{*}\right)}\right), \quad \mu \in(0,1),
$$

where $u(0)$ denotes $\left.u\right|_{x_{3}=0}$.

REMARK 1.1. The quantity $u(0) \in H^{2}\left(S_{*}\right)$ must be calculated independently. To this end let us consider problem (1.1) and let $G(x, y)$ be the Green function to (1.1) of the form

$$
G(x, y)=\frac{\vartheta(x, y)}{|x-y|}+g(x, y)
$$

where $\vartheta(x, y)$ is a smooth function such that

$$
\vartheta(x, y)= \begin{cases}1 & \text { for }|x-y| \leq 1 \\ 0 & \text { for }|x-y| \geq 2\end{cases}
$$

Then $g(x, y)$ is a solution to the problem

$$
\begin{aligned}
& -\Delta g=2 \nabla \frac{1}{|x-y|} \nabla \vartheta+\frac{1}{|x-y|} \Delta \vartheta, \\
& \left.\frac{\partial g}{\partial n}\right|_{S_{*}}=-\partial_{n} \frac{\vartheta}{|x-y|}, \\
& \left.g\right|_{S_{0} \cup S_{1}}=-\frac{\vartheta}{|x-y|},
\end{aligned}
$$

where $y \in \Omega$. Then any solution to (1.1) can be expressed as

$$
u(x)=\int_{\Omega} G(x, y) f(y) d y .
$$

Since $f=\alpha_{, x_{3}}$ (see 1.2 ) we obtain

$$
u(x)=\int_{S_{*}} G\left(x, y^{\prime}\right) \alpha\left(y^{\prime}\right) d y^{\prime}-\int_{\Omega} \partial_{y_{3}} G(x, y) \alpha(y) d y .
$$

Using the form of the Green function we have

$$
\|u(0)\|_{H^{2}\left(S_{*}\right)} \leq c\|\theta\|_{H^{1}\left(S_{*}\right)} .
$$

2. Estimates. Although the solution $u$ can be expressed by the Green function, we have to use another approach to show the properties of the solution described in the main theorem. Namely, to prove Theorem 1 we shall use local considerations.

Lemma 2.2. Assume that $f \in L_{2, \mu}(\Omega), \mu \in[0,1]$. Then there exists a solution to problem (1.1) such that

and

$$
u^{\prime}=u-u(0) \in H^{1}(\Omega) \cap L_{2,-\mu}(\Omega)
$$

$$
\left\|u^{\prime}\right\|_{H^{1}(\Omega)}+\left\|u^{\prime}\right\|_{L_{2,-\mu}(\Omega)} \leq c\left\|f^{\prime}\right\|_{L_{2, \mu}(\Omega)},
$$

where $u(0)=\left.u\right|_{x_{3}=0}$ and $f^{\prime}=f+\Delta u(0)$. 
Proof. First we obtain an energy type estimate. We reformulate (1.1) as follows:

$$
\begin{aligned}
& -\Delta u^{\prime}=f^{\prime} \quad \text { in } \Omega, \\
& \left.u_{, x_{3}}^{\prime}\right|_{S_{*}}=0, \\
& \left.u^{\prime}\right|_{S_{1}}=0, \\
& \left.u^{\prime}\right|_{S_{0}}=0 .
\end{aligned}
$$

Multiplying $2.2{ }_{1}$ by $u^{\prime}$, integrating by parts and using the boundary condition 2.2$)_{2}$ we have

$$
\begin{aligned}
\int_{\Omega}\left|\nabla u^{\prime}\right|^{2} d x & \leq \int_{\Omega} f^{\prime} u^{\prime} d x \\
& \leq\left(\int_{\Omega}\left|f^{\prime}\right|^{2} x_{3}^{2 \mu} d x\right)^{1 / 2}\left(\int_{\Omega}\left|u^{\prime}\right|^{2} x_{3}^{-2 \mu} d x\right)^{1 / 2} .
\end{aligned}
$$

We calculate

$$
u\left(x^{\prime}, x_{3}\right)-u\left(x^{\prime}, s\right)=\int_{s}^{x_{3}} \partial_{s^{\prime}} u\left(x^{\prime}, s^{\prime}\right) d s^{\prime},
$$

where $x^{\prime}=\left(x_{1}, x_{2}\right)$. By the Hölder inequality

$$
\frac{\left|u\left(x_{3}\right)-u(s)\right|}{\left|x_{3}-s\right|} \leq \int_{s}^{x_{3}}\left|\partial_{s^{\prime}} u\left(x^{\prime}, s^{\prime}\right)\right|^{2} d s^{\prime} .
$$

Setting $s=0$ and integrating the inequality over $\Omega^{\prime}=\left\{x \in \Omega: x_{3}=\right.$ const $\epsilon$ $[0, a]\}$ we have

$$
\int_{\Omega^{\prime}} \frac{\left|u\left(x_{3}\right)-u(0)\right|}{\left|x_{3}\right|} d x^{\prime} \leq \int_{\Omega^{\prime}} d x^{\prime} \int_{0}^{x_{3}}\left|\partial_{s^{\prime}} u\left(x^{\prime}, s^{\prime}\right)\right|^{2} d s^{\prime} .
$$

Now, we consider the second expression on the r.h.s. of 2.3$)$. We obtain

$$
\begin{aligned}
\int_{\Omega}\left|u^{\prime}\right|^{2} x_{3}^{-2 \mu} d x & \leq \int_{\Omega^{\prime}} d x^{\prime} \sup _{x_{3}} \frac{\left|u\left(x^{\prime}, x_{3}\right)-u(0)\right|^{2}}{x_{3}} \int_{0}^{a} \frac{d x_{3}}{x_{3}^{2 \mu-1}} \\
& \leq c \int_{\Omega^{\prime} 0}^{a}\left|\partial_{x_{3}} u\left(x^{\prime}, x_{3}\right)\right|^{2} d x_{3}
\end{aligned}
$$

where we used (2.4) and $\mu<1$.

Summarizing, we have

$$
\int_{\Omega}\left|u^{\prime}\right|^{2} x_{3}^{-2 \mu} d x+\int_{\Omega}\left|\nabla u^{\prime}\right|^{2} d x \leq c \int_{\Omega}\left|f^{\prime}\right|^{2} x_{3}^{2 \mu} d x, \quad \mu \in[0,1] .
$$

The existence of weak solutions to problem (1.1) satisfying (2.5) now follows from the Lax-Milgram theorem. 
To increase regularity of the weak solutions we consider problem 1.1 locally. Let $\zeta=\zeta\left(x^{\prime}\right)$ be a smooth function from a partition of unity. Introducing functions $\bar{u}=u \zeta, \bar{f}=f \zeta$ problem 1.1 takes the form

$$
\begin{aligned}
& \Delta \bar{u}=2 \nabla u \nabla \zeta+u \Delta \zeta+\bar{f} \equiv g, \\
& \left.\bar{u}\right|_{\partial \operatorname{supp} \zeta \cap\left\{x_{3}=a\right\}}=0, \\
& \left.\bar{u}_{, x_{3}}\right|_{\partial \operatorname{supp}} \zeta \cap\left\{x_{3}=0\right\} \\
& =0 .
\end{aligned}
$$

Suppose $\operatorname{supp} \zeta \cap S_{0} \neq \emptyset$. Assume that problem $(2.6)$ is written in local coordinates $x=\left(x_{1}, x_{2}, x_{3}\right)$ with origin in $\operatorname{supp} \zeta \cap S_{0}$. Now by using the local mapping $y=\Phi(x)$ such that $y_{3}=x_{3}$, we flatten the boundary $\operatorname{supp} \zeta \cap S_{0}$. Assume that in the new coordinates $y$ the flat boundary $\operatorname{supp} \zeta \cap S_{0}$ takes the form $y_{1}=0$. Let $v(y)=\bar{u}\left(\Phi^{-1}(y)\right), h(y)=g\left(\Phi^{-1}(y)\right)$. Then problem (2.6) takes the from

$$
\begin{aligned}
& \nabla_{y}^{2} v=\nabla_{y}^{2} v-\nabla_{\Phi}^{2} v+h \equiv k, \\
& \left.v\right|_{y_{1}=0}=0, \\
& \left.v\right|_{y_{3}=a}=0, \\
& \left.v_{x_{3}}\right|_{x_{3}=0}=0,
\end{aligned}
$$

where $\nabla_{\Phi}=\left.\frac{\partial y}{\partial x}\right|_{x=\Phi^{-1}(y)} \nabla_{y}$ and $v$ vanishes outside of $\left.\operatorname{supp} \zeta\right|_{x=\Phi^{-1}(y)}$.

Let us consider the reflection with respect to $y_{1}$ such that the reflected function $\mathbf{u}$ satisfies

$$
\begin{array}{ll}
\mathbf{u}\left(y_{1}, y_{2}, y_{3}\right)=v\left(y_{1}, y_{2}, y_{3}\right), & y_{1}>0 \\
\mathbf{u}\left(y_{1}, y_{2}, y_{3}\right)=v\left(-y_{1}, y_{2}, y_{3}\right), & y_{1}<0 .
\end{array}
$$

After the above reflection problem (2.7) takes the form

$$
\begin{array}{ll}
\nabla_{y}^{2} \mathbf{u}=\tilde{h} & \text { in } \mathbb{R}^{2} \times(0, a), \\
\left.\mathbf{u}\right|_{y_{3}=a}=0 & \text { on } \mathbb{R}^{2}, \\
\left.\mathbf{u}_{, y_{3}}\right|_{y_{3}=0}=0 & \text { on } \mathbb{R}^{2},
\end{array}
$$

where $\mathbf{u}$ has a compact support with respect to $y^{\prime}$.

If $\operatorname{supp} \zeta \cap S_{0}=\emptyset$ we can extend problem $(2.6)$ by zero with respect to $x^{\prime}=\left(x_{1}, x_{2}\right)$. Then problem 2.6 can also be expressed as 2.8.

Let us consider the Fourier transform

$$
\hat{u}\left(\xi, x_{3}\right)=\int_{\mathbb{R}^{2}} e^{-i x^{\prime} \cdot \xi} \mathbf{u}\left(x^{\prime}, x_{3}\right) d x^{\prime},
$$

where $\xi=\left(\xi_{1}, \xi_{2}\right), x^{\prime} \cdot \xi=x_{1} \xi_{1}+x_{2} \xi_{2}$. Applying this transformation to problem 2.8), where $\tilde{h}$ is replaced by $f$ and $y$ by $x$, we obtain, in the domain 
$\widehat{\Omega}=\left\{\left(x^{\prime}, x_{3}\right): x^{\prime} \in \mathbb{R}^{2}, 0 \leq x_{3} \leq a\right\}$, the problem

$$
\begin{aligned}
& -\frac{d^{2} \hat{u}}{d x_{3}^{2}}+\xi^{2} \hat{u}=\hat{f}, \\
& \left.\hat{u}\right|_{x_{3}=a}=0, \\
& \left.\hat{u}_{x_{3}}\right|_{x_{3}=0}=0 .
\end{aligned}
$$

We can solve this problem explicitly:

Lemma 2.3. Problem (2.9) has the solution

$$
\begin{aligned}
\hat{u}= & \beta_{0} \cosh \left(|\xi| x_{3}\right)-\int_{0}^{x_{3}} \frac{\cosh (|\xi| \tau) \hat{f}(\xi, \tau)}{|\xi|} d \tau \sinh \left(|\xi| x_{3}\right) \\
& +\int_{0}^{x_{3}} \frac{\sinh (|\xi| \tau) \hat{f}(\xi, \tau)}{|\xi|} d \tau \cosh \left(|\xi| x_{3}\right)
\end{aligned}
$$

where

$$
\beta_{0}=\int_{0}^{a} \frac{\cosh (|\xi| \tau) \hat{f}(\xi, \tau)}{|\xi|} d \tau \frac{\sinh (|\xi| a)}{\cosh (|\xi| a)}-\int_{0}^{a} \frac{\sinh (|\xi| \tau) \hat{f}(\xi, \tau)}{|\xi|} d \tau
$$

Proof. General solutions of homogeneous equations $(2.9)_{1}$ have the form

$$
\hat{u}=\alpha \sinh \left(|\xi| x_{3}\right)+\beta \cosh \left(|\xi| x_{3}\right) .
$$

We can find solutions to 2.9 by variation of constants. We have the following equations for $\alpha\left(x_{3}\right), \beta\left(x_{3}\right)$ :

$$
\begin{aligned}
& \frac{d \alpha}{d x_{3}} \sinh \left(|\xi| x_{3}\right)+\frac{d \beta}{d x_{3}} \cosh \left(|\xi| x_{3}\right)=0 \\
& \frac{d \alpha}{d x_{3}} \cosh \left(|\xi| x_{3}\right)+\frac{d \beta}{d x_{3}} \sinh \left(|\xi| x_{3}\right)=-\frac{\hat{f}}{|\xi|} .
\end{aligned}
$$

Solving this yields

Hence, we get

$$
\frac{d \alpha}{d x_{3}}=-\frac{\cosh \left(|\xi| x_{3}\right)}{|\xi|} \hat{f}, \quad \frac{d \beta}{d x_{3}}=\frac{\sinh \left(|\xi| x_{3}\right)}{|\xi|} \hat{f} .
$$

$$
\alpha=-\int_{0}^{x_{3}} \frac{\cosh (|\xi| \tau) \hat{f}(\xi, \tau)}{|\xi|} d \tau, \quad \beta=\int_{0}^{x_{3}} \frac{\sinh (|\xi| \tau) \hat{f}(\xi, \tau)}{|\xi|} d \tau .
$$

Using the formulas for $\alpha, \beta$ in 2.12 we postulate the general solution of 2.9

(2.13) $\hat{u}=\alpha_{0} \sinh \left(|\xi| x_{3}\right)+\beta_{0} \cosh \left(|\xi| x_{3}\right)-\int_{0}^{x_{3}} \frac{\cosh (|\xi| \tau) \hat{f}(\xi, \tau)}{|\xi|} d \tau \sinh \left(|\xi| x_{3}\right)$

$$
+\int_{0}^{x_{3}} \frac{\sinh (|\xi| \tau) \hat{f}(\xi, \tau)}{|\xi|} d \tau \cosh \left(|\xi| x_{3}\right)
$$


The boundary conditions $(2.9)_{2-3}$ imply the following equations for $\alpha_{0}$ and $\beta_{0}$ :

$$
\begin{aligned}
& \alpha_{0} \sinh (|\xi| a)+\beta_{0} \cosh (|\xi| a)-\int_{0}^{a} \frac{\cosh (|\xi| \tau) \hat{f}(\xi, \tau)}{|\xi|} d \tau \sinh (|\xi| a) \\
& +\int_{0}^{a} \frac{\sinh (|\xi| \tau) \hat{f}(\xi, \tau)}{|\xi|} d \tau \cosh (|\xi| a)=0, \\
& -\alpha_{0}|\xi|=0 .
\end{aligned}
$$

Solving (2.14) with respect to $\alpha_{0}, \beta_{0}$ implies formula (2.11) with $\alpha_{0}=0$, and inserting the result in the general solution formula 2.13) we obtain 2.10). This concludes the proof.

COROLlary 2.1. The function $\hat{u}$ given by formula (2.10) is the unique solution to problem 2.9).

Now we obtain an estimate for the solution to problem (2.9), given by 2.10 . We set $\left.\hat{u}\right|_{x_{3}=0}=\hat{u}(0)$ and $\hat{u}-\hat{u}(0) \equiv \breve{u}$.

Lemma 2.4. Assume that

$$
\begin{aligned}
& \int_{\mathbb{R}^{2}} d \xi \int_{(0, a)}|\hat{f}|^{2} x_{3}^{2 \mu} d x_{3}<\infty, \\
& \int_{\mathbb{R}^{2}} \xi^{4} d \xi\|\hat{u}(0)\|_{L_{2, \mu}(0, a)}^{2}<\infty, \\
& \int_{\mathbb{R}^{2}} \xi^{2} d \xi \int_{(0, a)}|\breve{u}|^{2} x_{3}^{2 \mu-2} d x_{3}<\infty .
\end{aligned}
$$

Then the solution $\hat{u}$ to the problem (2.9) satisfies

$$
\begin{array}{r}
\int_{\mathbb{R}^{2}} \xi^{2} d \xi \int_{(0, a)}\left(\left|\breve{u}_{, x_{3}}\right|^{2}+\xi^{2}|\breve{u}|^{2}\right) x_{3}^{2 \mu} d x_{3}+\int_{\mathbb{R}^{2}} d \xi\left(\left\|\partial_{x_{3}}^{2} \breve{u}\right\|_{L_{2, \mu}(0, a)}^{2}\right. \\
\left.+\left\|\partial_{x_{3}} \breve{u}\right\|_{L_{2, \mu-1}(0, a)}^{2}+\|\breve{u}\|_{L_{2, \mu-2}\left(\mathbb{R}^{2}\right)}^{2}\right) \\
\leq c_{1} \int_{\mathbb{R}^{2}} \xi^{2} d \xi \int_{(0, a)}|\breve{u}|^{2} x_{3}^{2 \mu-2} d x_{3}+c \int_{\mathbb{R}^{2}} d \xi \int_{(0, a)}|\hat{f}|^{2} x_{3}^{2 \mu} d x_{3} \\
+c \int_{\mathbb{R}^{2}} \xi^{4} d \xi\|\hat{u}(0)\|_{L_{2, \mu}(0, a)}^{2}
\end{array}
$$

Proof. Multiplying 2.9$)_{1}$ by $\bar{u} x_{3}^{2 \mu}$ and integrating on $(0, a)$ we get

$$
\int_{(0, a)}\left(-\hat{u}_{, x_{3} x_{3}} \overline{\breve{u}}+\xi^{2}|\breve{u}|^{2}\right) x_{3}^{2 \mu} d x_{3}=\int_{(0, a)} \hat{f} \bar{u} x_{3}^{2 \mu} d x_{3}-\xi^{2} \int_{(0, a)} \hat{u}(0) \overline{\breve{u}} x_{3}^{2 \mu} d x_{3} .
$$


Integrating by parts we obtain

$$
\begin{aligned}
\int_{(0, a)}\left(\left|\breve{u}_{, x_{3}}\right|^{2} x_{3}^{2 \mu}+\xi^{2}|\breve{u}|^{2} x_{3}^{2 \mu}\right) d x_{3} & \\
= & -2 \mu \int_{(0, a)} \hat{u}, x_{3} \overline{\breve{u}} x_{3}^{2 \mu-1} d x_{3} \\
& +\int_{(0, a)} \hat{f} \bar{u} x_{3}^{2 \mu} d x_{3}-\xi^{2} \int_{(0, a)} \hat{u}(0) \bar{u} x_{3}^{2 \mu} d x_{3} .
\end{aligned}
$$

By the Hölder and Young inequalities we estimate the first term on the r.h.s. of 2.17) by

$$
\frac{\varepsilon_{1}}{2} \int_{(0, a)}\left|\breve{u}_{, x_{3}}\right|^{2} x_{3}^{2 \mu} d x_{3}+\frac{4 \mu^{2}}{2 \varepsilon_{1}} \int_{(0, a)}|\breve{u}|^{2} x_{3}^{2 \mu-2} d x_{3},
$$

the second by

$$
\frac{\varepsilon_{2}}{2} \xi^{2} \int_{(0, a)}|\breve{u}|^{2} x_{3}^{2 \mu} d x_{3}+\frac{1}{2 \varepsilon_{2}} \frac{1}{\xi^{2}} \int_{(0, a)}|\hat{f}|^{2} x_{3}^{2 \mu} d x_{3}
$$

and the third by

$$
\frac{\varepsilon_{3}}{2} \xi^{2} \int_{(0, a)}|\breve{u}|^{2} x_{3}^{2 \mu} d x_{3}+\frac{1}{2 \varepsilon_{3}} \xi^{2} \int_{(0, a)}|\hat{u}(0)|^{2} x_{3}^{2 \mu} d x_{3} .
$$

Setting $\varepsilon_{1}=1, \varepsilon_{2}=\varepsilon_{3}=1 / 2$, we obtain from 2.17 the inequality

$$
\begin{aligned}
\frac{1}{2} \int_{(0, a)}\left(\left|\breve{u}_{, x_{3}}\right|^{2}+\xi^{2}|\breve{u}|^{2}\right) x_{3}^{2 \mu} d x_{3} \leq & 2 \mu^{2} \int_{(0, a)}|\breve{u}|^{2} x_{3}^{2 \mu-2} d x_{3}+\frac{1}{\xi^{2}} \int_{(0, a)}|\hat{f}|^{2} x_{3}^{2 \mu} d x_{3} \\
& +\xi^{2} \int_{(0, a)}|\hat{u}(0)|^{2} x_{3}^{2 \mu} d x_{3} .
\end{aligned}
$$

We multiply this by $2 \xi^{2}$ and integrate with respect to $\xi$ to get

$$
\begin{aligned}
\int_{\mathbb{R}^{2}} \xi^{2} d \xi \int_{(0, a)}\left(\left|\breve{u}_{, x_{3}}\right|^{2}+\xi^{2}|\breve{u}|^{2}\right) x_{3}^{2 \mu} d x_{3} \leq 4 \mu^{2} \int_{\mathbb{R}^{2}} \xi^{2} d \xi \int_{(0, a)}|\breve{u}|^{2} x_{3}^{2 \mu-2} d x_{3} \\
+2 \int_{\mathbb{R}^{2}} d \xi \int_{(0, a)}|\hat{f}|^{2} x_{3}^{2 \mu} d x_{3}+2 \int_{\mathbb{R}^{2}} \xi^{4} d \xi \int_{(0, a)}|\hat{u}(0)|^{2} x_{3}^{2 \mu} d x_{3} .
\end{aligned}
$$

This yields an estimate for the first integral in (2.16). To deal with the other terms, we slightly reformulate problem $(2.9)$ to the form

$$
\begin{aligned}
& \frac{d^{2} \hat{u}}{d x_{3}^{2}}=\xi^{2} \hat{u}-\hat{f}, \\
& \left.\hat{u}\right|_{x_{3}=a}=0, \\
& \left.\hat{u}_{x_{3}}\right|_{x_{3}=0}=0 .
\end{aligned}
$$


We derive the bound

$$
\left\|\partial_{x_{3}}^{2}(\breve{u})\right\|_{L_{2, \mu}(0, a)}^{2} \leq \xi^{4}\|\breve{u}\|_{L_{2, \mu}(0, a)}^{2}+\xi^{4}\|\hat{u}(0)\|_{L_{2, \mu}(0, a)}^{2}+\|\hat{f}\|_{L_{2, \mu}(0, a)} .
$$

Consequently, integrating with respect to $\xi$ implies

$$
\begin{aligned}
\int_{\mathbb{R}^{2}} d \xi\left\|\partial_{x_{3}}^{2}(\breve{u})\right\|_{L_{2, \mu}(0, a)}^{2} & \leq \int_{\mathbb{R}^{2}} \xi^{4} d \xi\|\breve{u}\|_{L_{2, \mu}(0, a)}^{2} \\
& +\int_{\mathbb{R}^{2}} \xi^{4} d \xi\|\hat{u}(0)\|_{L_{2, \mu}(0, a)}^{2}+\int_{\mathbb{R}^{2}} d \xi\|\hat{f}\|_{L_{2, \mu}(0, a)} .
\end{aligned}
$$

On the other hand, by the Hardy inequality we have

$$
\begin{aligned}
\int_{\mathbb{R}^{2}} d \xi\left\|\partial_{x_{3}}^{2}(\breve{u})\right\|_{L_{2, \mu}(0, a)}^{2} & \geq c \int_{\mathbb{R}^{2}} d \xi\left\|\partial_{x_{3}}(\breve{u})\right\|_{L_{2, \mu-1}(0, a)}^{2} \\
& \geq c \int_{\mathbb{R}^{2}} d \xi\|\breve{u}\|_{L_{2, \mu-2}(0, a)}^{2}
\end{aligned}
$$

and this with 2.20 gives an estimate for the second integral on the 1.h.s. of (2.16). Therefore, combining 2.18), 2.20 and 2.21) we derive 2.16), which concludes the proof.

Next, we need to improve the lemma dealing with the term on the r.h.s. of 2.16 involving $\breve{u}$. To this end, we introduce the sets:

$$
\begin{aligned}
& Q_{1}=\left\{\left(\xi, x_{3}\right) \in \mathbb{R}^{2} \times \mathbb{R}_{+}:|\xi|^{-1} x_{3}^{-1} \leq a_{1}\right\}, \\
& Q_{2}=\left\{\left(\xi, x_{3}\right) \in \mathbb{R}^{2} \times \mathbb{R}_{+}:|\xi|^{-1} x_{3}^{-1} \geq a_{2}\right\}, \\
& Q_{3}=\left\{\left(\xi, x_{3}\right) \in \mathbb{R}^{2} \times \mathbb{R}_{+}: a_{1} \leq|\xi|^{-1} x_{3}^{-1} \leq a_{2}\right\},
\end{aligned}
$$

and prove the following result:

LEMMA 2.5. Let assumptions 2.15 1-2 of Lemma 2.3 be satisfied. Then for a solution $\hat{u}$ to problem 2.9 and $\vec{u}=\hat{u}-\hat{u}(0)$,

$$
\begin{aligned}
& \text { (2.22) } \int_{\mathbb{R}^{2}} \xi^{2} d \xi \int_{(0, a)}|\breve{u}|^{2} x_{3}^{2 \mu-2} d x_{3} \leq a_{1}^{2} \int_{Q_{1}} \xi^{4}|\breve{u}|^{2} x_{3}^{2 \mu} d \xi d x_{3} \\
& +\frac{1}{a_{2}^{2}} \int_{Q_{2}}|\breve{u}|^{2} x_{3}^{2 \mu-4} d \xi d x_{3}+c \int_{\mathbb{R}^{2} \times(0, a)} \xi^{4}|\hat{u}(0)|^{2} x_{3}^{2 \mu} d \xi d x_{3}+c \int_{\mathbb{R}^{2} \times(0, a)}|\hat{f}|^{2} x_{3}^{2 \mu} d \xi d x_{3} .
\end{aligned}
$$

Proof. Let us consider the expression

$$
\int_{\mathbb{R}^{2}} \xi^{2} d \xi \int_{(0, a)}|\breve{u}|^{2} x_{3}^{2 \mu-2} d x_{3}=\sum_{i=1}^{3} \int_{Q_{i}} \xi^{2}|\breve{u}|^{2} x_{3}^{2 \mu-2} d \xi d x_{3}=\sum_{i=1}^{3} I_{i}
$$

where 


$$
\begin{aligned}
& I_{1} \leq a_{1}^{2} \int_{Q_{1}} \xi^{4}|\breve{u}|^{2} x_{3}^{2 \mu} d \xi d x_{3}, \\
& I_{2} \leq \frac{1}{a_{2}^{2}} \int_{Q_{2}}|\breve{u}|^{2} x_{3}^{2 \mu-4} d \xi d x_{3}, \\
& I_{3} \leq a_{2}^{2-2 \mu} \int_{Q_{3}}|\xi|^{4-2 \mu}|\breve{u}|^{2} d \xi d x_{3} .
\end{aligned}
$$

Let us express problem 2.19 in the form

$$
\partial_{x_{3}}^{2} \breve{u}=\xi^{2} \breve{u}-\hat{f}+\xi^{2} \hat{u}(0) .
$$

Then from [ZS] and [Z1, (4.12)] we extract the inequality

$$
\begin{aligned}
I_{3} & \leq c \int_{Q_{3}}|\xi|^{4-2 \mu}|\breve{u}|^{2} d \xi d x_{3} \\
& \leq c \int_{\mathbb{R}^{2} \times(0, a)} \xi^{4}|\hat{u}(0)|^{2} x_{3}^{2 \mu} d \xi d x_{3}+c \int_{\mathbb{R}^{2} \times(0, a)}|\hat{f}|^{2} x_{3}^{2 \mu} d \xi d x_{3} .
\end{aligned}
$$

Collecting the estimates for $I_{i}$ we obtain 2.22 , and this concludes the proof.

Corollary 2.2. Consider the solution $\hat{u}$ of 2.9). For sufficiently small $a_{1}$ and sufficiently large $a_{2}$, assuming 2.15 ${ }_{1-2}$, estimates 2.16 and 2.22 imply

$$
\begin{aligned}
\int_{\mathbb{R}^{2}} d \xi\|\breve{u}\|_{H_{\mu}^{2}(0, a)}^{2} & +\int_{\mathbb{R}^{2}} \xi^{2} d \xi\|\breve{u}\|_{H_{\mu}^{1}(0, a)}^{2}+\int_{\mathbb{R}^{2}} \xi^{4} d \xi\|\breve{u}\|_{L_{2, \mu}(0, a)}^{2} \\
& \leq c \int_{\mathbb{R}^{2}} d \xi\|\hat{f}\|_{L_{2, \mu}(0, a)}^{2}+c \int_{\mathbb{R}^{2}} \xi^{4} d \xi\|\hat{u}(0)\|_{L_{2, \mu}(0, a)}^{2}
\end{aligned}
$$

Next, we want to estimate the last term in 2.23 . It takes the form

$$
\int_{\mathbb{R}^{2} \times(0, a)}\left|\partial_{x^{\prime}}^{2} u(0)\right|^{2}\left|x_{3}\right|^{2 \mu} d x^{\prime} d x_{3} \leq c \int_{\mathbb{R}^{2}}\left|\partial_{x^{\prime}}^{2} u(0)\right|^{2} d x^{\prime},
$$

where we used the fact that the support of $u$ with respect to $x_{3}$ is compact.

Now we postulate that the function $f$ in 1.1 can be written as

$$
f=\alpha_{, x_{3}} \quad \text { where } \quad \alpha=\tilde{a} \eta\left(x_{3}\right),\left.\tilde{a}\right|_{x_{3}=0}=\theta
$$

and $\eta\left(x_{3}\right)$ is a smooth non-increasing function with compact support, $0 \leq$ $\eta \leq 1$ and $\eta\left(x_{3}\right)=1$ in a neighbourhood of $\left\{x_{3}=0\right\}$. We extend the function $\theta$ onto $\mathbb{R}^{2}$ in $H^{1}\left(\mathbb{R}^{2}\right)$ norm using the Hestenes-Whitney theorem in such a way that

$$
\left.\tilde{\theta}\right|_{S_{*}}=\theta, \quad\|\tilde{\theta}\|_{H^{k}\left(\mathbb{R}^{2}\right)} \leq c\|\theta\|_{H^{k}\left(S_{*}\right)}
$$


Then we solve the problem

$$
\begin{aligned}
& \Delta u=\alpha_{, x_{3}}, \quad x_{3}>0, \\
& \left.u_{, x_{3}}\right|_{x_{3}=0}=0 .
\end{aligned}
$$

Lemma 2.6. Assume that $u$ is a solution to 2.26), where $\alpha$ is described by $(1.2)$. Assume that $\theta \in H^{1}\left(S_{*}\right)$. Then

$$
\left\|\partial_{x^{\prime}}^{2} u(0)\right\|_{L^{2}\left(\mathbb{R}^{2}\right)} \leq c\|\theta\|_{H^{1}\left(S_{*}\right)} .
$$

Proof. Using the Neumann function, any solution to 2.26 can be expressed by

$$
u(x)=\int_{\mathbb{R}_{+}^{3}}\left(\frac{1}{|x-y|}+\frac{1}{|x-\bar{y}|}\right) \alpha_{, y_{3}} d y
$$

where $\bar{y}=\left(y_{1}, y_{2},-y_{3}\right), \mathbb{R}_{+}^{3}=\left\{x \in \mathbb{R}^{3}: x_{3}>0\right\}$.

Integrating by parts we obtain

$$
\begin{aligned}
u(x) & =\int_{\mathbb{R}_{+}^{3}} \partial_{y_{3}}\left[\left(\frac{1}{|x-y|}+\frac{1}{|x-\bar{y}|}\right) \alpha\right] d y-\int_{\mathbb{R}_{+}^{3}} \partial_{y_{3}}\left(\frac{1}{|x-y|}+\frac{1}{|x-\bar{y}|}\right) \alpha d y \\
& =\int_{\mathbb{R}^{2}}\left(\frac{1}{|x-y|}+\frac{1}{|x-\bar{y}|}\right) \mid y_{3}=0 \tilde{\theta} d y^{\prime}-\int_{\mathbb{R}_{+}^{3}}\left(\frac{x_{3}-y_{3}}{|x-y|^{3}}-\frac{x_{3}+y_{3}}{|x-\bar{y}|^{3}}\right) \tilde{a} \eta d y \\
& =\int_{\mathbb{R}^{2}} \frac{2}{\sqrt{\left|x^{\prime}-y^{\prime}\right|^{2}+x_{3}^{2}}} \tilde{\theta} d y^{\prime}-\int_{\mathbb{R}_{+}^{3}}\left(\frac{x_{3}-y_{3}}{|x-y|^{3}}-\frac{x_{3}+y_{3}}{|x-\bar{y}|^{3}}\right) \tilde{a} \eta d y .
\end{aligned}
$$

In view of this formula, the r.h.s. of (2.24) assumes the form

$$
\begin{aligned}
& \int_{\mathbb{R}^{2}}\left|\partial_{x^{\prime}}^{2} u(0)\right|^{2} d x^{\prime} \\
& \quad \leq 4 \int_{\mathbb{R}^{2}} d x^{\prime}\left|\partial_{x^{\prime}}^{2} \int_{\mathbb{R}^{2}} \frac{1}{\left|x^{\prime}-y^{\prime}\right|} \tilde{\theta} d y^{\prime}\right|^{2}+4 \int_{\mathbb{R}^{2}} d x^{\prime}\left|\partial_{x^{\prime}}^{2} \int_{\mathbb{R}_{+}^{3}} \frac{y_{3}}{\sqrt{\left|x^{\prime}-y^{\prime}\right|^{2}+y_{3}^{2}}} \tilde{\theta} d y_{3}\right|^{2} \\
& \quad=4 \int_{\mathbb{R}_{\tilde{N}}^{2}} d x^{\prime}\left|\int_{\mathbb{R}^{2}} \partial_{x^{\prime}}^{2} \frac{1}{\left|x^{\prime}-y^{\prime}\right|} \tilde{\theta} d y^{\prime}\right|^{2}+4 \int_{\mathbb{R}^{2}} d x^{\prime} \mid \int_{\mathbb{R}^{2}} \partial_{x^{\prime}}^{2} \frac{y_{3}}{\left.\sqrt{\left|x^{\prime}-y^{\prime}\right|^{2}+y_{3}^{2}} d y_{3}\right|^{2}} \\
& \quad \leq c\|\|_{H^{1}\left(\mathbb{R}^{2}\right)} \leq c\|\theta\|_{H^{1}\left(S_{*}\right)} .
\end{aligned}
$$

This concludes the proof.

Using (2.23), (2.27) and applying a partition of unity for $\Omega$ we deduce the following result:

Lemma 2.7. For problem 1.1), where $f \in L_{2, \mu}\left(\mathbb{R}^{2} \times(0, a)\right), f$ is expressed by $(1.2)$ and $\theta \in H^{1}\left(\mathbb{R}^{2}\right)$, the solution $u$ satisfies (2.28) $\quad\|u-u(0)\|_{H_{\mu}^{2}\left(\mathbb{R}^{2} \times(0, a)\right)} \leq c\left(\|f\|_{L_{2, \mu}\left(\mathbb{R}^{2} \times(0, a)\right)}+\|\theta\|_{H^{1}\left(\mathbb{R}^{2}\right)}\right)$, where $u(0)$ denotes $\left.u\right|_{x_{3}=0}$. 
Proof of Theorem 1. We apply the regularizer technique for elliptic equations (see [S], [LSU]). Let us define two collections of open subsets $\left\{\omega^{(k)}\right\}$ and $\left\{\Omega^{(k)}\right\}, k \in \mathcal{M} \cup \mathcal{N}$, such that $\overline{\omega^{(k)}} \subset \Omega^{(k)}, \bigcup_{k} \omega^{(k)}=\bigcup_{k} \Omega^{(k)}=\Omega \cap P$, where $P$ is any plane perpendicular to the $x_{3}$ axis. We assume that $\omega^{(k)} \cap S=\emptyset$ for $k \in \mathcal{M}$ and $\omega^{(k)} \cap S \neq \emptyset$ for $k \in \mathcal{N}$. Then $\bigcup_{k} \omega^{(k)} \times(0, a)=\bigcup_{k} \Omega^{(k)} \times(0, a)=\Omega$.

Let $\zeta^{(k)}\left(x^{\prime}\right)$ be a smooth function such that $0 \leq \zeta^{(k)}\left(x^{\prime}\right) \leq 1, \zeta^{(k)}\left(x^{\prime}\right)=1$ for $x^{\prime} \in \omega^{(k)}, \operatorname{supp} \zeta^{(k)} \subset \Omega^{(k)}$ and $\left|D_{x^{\prime}}^{\nu} \zeta^{(k)}\left(x^{\prime}\right)\right| \leq(c /|\lambda|) \nu$, where $\lambda$ is the diameter of $\Omega$. Then $1 \leq \sum_{k} \zeta^{(k)}\left(x^{\prime}\right) \leq N_{0}$. Introducing the function $\eta^{(k)}\left(x^{\prime}\right)=$ $\zeta^{(k)}\left(x^{\prime}\right) / \sum_{l}\left(\zeta^{(l)}\left(x^{\prime}\right)\right)^{2}$ we have $\operatorname{supp} \eta^{(k)}\left(x^{\prime}\right) \subset \Omega^{(k)}, \sum_{k} \eta^{(k)}\left(x^{\prime}\right) \zeta^{(k)}\left(x^{\prime}\right)=1$, $\left|D_{x^{\prime}}^{\nu} \eta^{(k)}\left(x^{\prime}\right)\right| \leq c /|\lambda|^{\nu}$.

By $\xi^{(k)}$ we denote a fixed internal point of $\omega^{(k)}$ and $\Omega^{(k)}$ for $k \in \mathcal{M}$ and a point of $\overline{\omega^{(k)}} \cap S$ and $\overline{\Omega^{(k)}} \cap S$ for $k \in \mathcal{N}$. Let us introduce a local coordinate system $y=\left(y_{1}, y_{2}\right)$ with centre at $\xi^{(k)}, k \in \mathcal{N}$. We assume that $y_{2}=F\left(y_{1}\right)$ describes the part $S^{(k)}=S \cap \overline{\Omega^{(k)}}$ of the boundary. Let us introduce new coordinates by

$$
z_{1}=y_{1}, \quad z_{2}=y_{2}-F\left(y_{1}\right) .
$$

Let $\Psi_{k}$ denote the transformation

$$
\Omega^{(k)} \ni y^{\prime} \mapsto \Psi_{k}\left(y^{\prime}\right)=z^{\prime} \in \hat{\Omega}^{(k)}, \quad \omega^{(k)} \ni y^{\prime} \mapsto \Phi_{k}\left(y^{\prime}\right)=z^{\prime} \in \hat{\omega}^{(k)},
$$

where $\hat{\omega}^{(k)}$ and $\hat{\Omega}^{(k)}$ are described by the relations

$$
\begin{aligned}
\left|y_{1}\right|<\lambda, & 0<y_{2}-F\left(y_{1}\right)<\lambda, \\
\left|y_{1}\right|<2 \lambda, & 0<y_{2}-F\left(y_{1}\right)<2 \lambda,
\end{aligned}
$$

respectively. Let $y=Y_{k}(x)$ be a transformation from global coordinates $x$ to local coordinates with origin at $\xi^{(k)}$ which is a composition of a translation and a rotation. We denote $\Phi_{k}=\Psi_{k} \cdot Y_{k}$. We set

$$
\hat{u}^{(k)}(z)=u\left(\Phi_{k}^{-1}(z)\right), \quad \tilde{u}^{(k)}(z)=\hat{u}^{(k)}(z) \hat{\zeta}^{(k)}(z) .
$$

For $k \in \mathcal{M}$ problem 1.1 translates into the equation

$$
-\nabla_{x}^{2} \tilde{u}^{(k)}=\tilde{f}_{*}^{(k)} \quad \text { in } \mathbb{R}^{2} \times(0, a)
$$

and for $k \in \mathcal{N}$ into the equation

$$
-\nabla_{z}^{2} \tilde{u}^{(k)}=\tilde{f}_{*}^{(k)} \quad \text { in } \mathbb{R}^{2} \times(0, a),
$$

which is appropriately extended onto $z_{2}<0$. Let $R^{(k)}$ be the operator which solves problems (2.31) and (2.32), respectively. Then we define the operator

$$
R f=\sum_{k \in \mathcal{M} \cup \mathcal{N}} \eta^{(k)} u^{(k)}
$$


where

$$
u^{(k)}(x)= \begin{cases}R^{(k)} \zeta^{(k)}, & k \in \mathcal{M} \\ \Phi_{k}^{-1}\left(\Phi_{k}^{-1} R^{(k)}\left(\Phi_{k} \zeta^{(k)} f\right)\right), & k \in \mathcal{N}\end{cases}
$$

The solvability of problems 2.31) and 2.32 is settled by Lemma 2.4 .

Let us introduce spaces $H=L_{2, \mu}, V=H_{\mu}^{2}$. Let $\mathcal{L}=-\Delta$. Then we examine operators $T, W$, where

$$
\mathcal{L} R f=(I+T) f, \quad R \mathcal{L} v=(I+W) v .
$$

We calculate

$$
\begin{aligned}
T f= & \sum_{k \in \mathcal{M} \cup \mathcal{N}}\left(\mathcal{L} \eta^{(k)} u^{(k)}-\eta^{(k)} \mathcal{L} u^{(k)}\right) \\
& +\sum_{k \in \mathcal{N}} \eta^{(k)} \Phi_{k}^{-1}\left[\mathcal{L}\left(\partial_{z}-\nabla F \partial_{z_{2}}\right)-\mathcal{L}\left(\partial_{z}\right)\right] R^{(k)}\left(\Phi_{k} \zeta^{(k)} f\right) \\
= & T_{1}+T_{2}
\end{aligned}
$$

and

$$
\begin{aligned}
T_{1} f= & \sum_{k \in \mathcal{M}} \eta^{(k)} \Phi_{k}^{-1}\left[-\partial_{z_{1}} F \partial_{z_{1}} F \partial_{z_{2}}^{2}-\partial_{z_{1}} F \partial_{z_{1}} \partial_{z_{2}}\right. \\
& \left.-\partial_{z_{1}} F \partial_{z_{2}} \partial_{z_{1}}-\partial_{z_{1}} F \partial_{z_{1} z_{2}} F \partial_{z_{2}}\right] R^{(k)}\left(\Phi_{k} \zeta^{(k)} f\right) \\
T_{2} f= & \sum_{k \in \mathcal{M} \cup \mathcal{N}}\left(\mathcal{L} \eta^{(k)} u^{(k)}-\eta^{(k)} \mathcal{L} u^{(k)}\right) \\
& +\sum_{k \in \mathcal{N}} \eta^{(k)} \Phi_{k}^{-1}\left(-\partial_{z_{1}}^{2} F \partial_{z_{2}}\right) R^{(k)}\left(\Phi_{k} \zeta^{(k)} f\right) .
\end{aligned}
$$

Since $\left|\partial_{z_{1}} F\right| \leq c \lambda$, we have

$$
\left\|T_{1} f\right\|_{H} \leq c \lambda\|f\|_{H}
$$

so $\left\|T_{1}\right\|_{H} \leq 1$ for sufficiently small $\lambda$. On the other hand $T_{2}$ is completely continuous. Similarly, $W=W_{1}+W_{2}$, where

$W_{1} u$

$$
=\sum_{k \in \mathcal{N}} \eta^{(k)} \Phi_{k}^{-1} R^{(k)}\left[-\partial_{z_{1}} F \partial_{z_{1}} F \partial_{z_{2}}^{2}-2 \partial_{z_{1}} F \partial_{z_{1} z_{2}}-\partial_{z_{1}} F \partial_{z_{2}}\left(\partial_{z_{1}} F\right) \partial_{z_{2}}\right] \Phi_{k} \zeta^{(k)} u
$$

and

$$
\begin{aligned}
W_{2} u= & \sum_{k \in \mathcal{M}} \eta^{(k)} R^{(k)}\left(\zeta^{(k)} \mathcal{L}-\mathcal{L} \zeta^{(k)}\right) u+\sum_{k \in \mathcal{N}} \eta^{(k)} \Phi_{k}^{-1} R^{(k)}\left[\Phi_{k}\left(\zeta^{(k)} \mathcal{L}-\mathcal{L} \zeta^{(k)}\right) u\right] \\
& -\sum_{k \in \mathcal{N}} \eta^{(k)} \Phi_{k}^{-1} R^{(k)}\left(F_{z_{1} z_{1}} \partial_{z_{2}}\right) \Phi_{k} \zeta^{(k)} u .
\end{aligned}
$$

By the same arguments as above we have $\left\|W_{1}\right\|_{V}<1$ and $W_{2}$ is compact and continuous. 
We write 2.33$)_{2}$ in the form

$$
\left(I+W_{1}\right) v=R f+W_{2} v .
$$

Here $R$ is a bounded operator from $H_{\mu}^{2}$ in $L_{2, \mu}, W_{2}$ is a completely continuous operator with

$$
\left\|W_{2}\right\|_{H_{\mu}^{2}(\Omega)} \leq \varepsilon\|v\|_{H_{\mu}^{2}(\Omega)}+c(1 / \varepsilon)\|v\|_{L_{2}(\Omega)}
$$

and $W_{1}$ is the operator with norm less than one. Then from (2.34) we obtain

$$
\|v\|_{H_{\mu}^{2}(\Omega)} \leq c\|\mathcal{L} v\|_{H_{\mu}^{2}(\Omega)}+c\|v\|_{L_{2}(\Omega)},
$$

where by using the Green function and the Hardy inequality we find that

$$
\|v\|_{L_{2}(\Omega)} \leq c\|\alpha\|_{L_{2}(\Omega)} \leq c\|f\|_{L_{2, \mu}(\Omega)} .
$$

We have

$$
\|v\|_{H_{\mu}^{2}(\Omega)} \leq c\|\mathcal{L} v\|_{L_{2, \mu}(\Omega)} .
$$

Hence there exists an inverse operator to $\mathcal{L}$ so we have existence in $H_{\mu}^{2}(\Omega)$.

Acknowledgments. This research was partially supported by MNiSW grant no 1 P03A 02130 and EC FP6 Marie Curie ToK programme SPADE2.

\section{References}

[CF] R. Coifman and C. Fefferman, Weighted norm inequalities for maximal functions and singular integrals, Studia Math. 51 (1974), 241-250.

[DS] N. Dunford and J. T. Schwartz, Linear Operators, Interscience, 1966.

[G] G. P. Galdi, An Introduction to the Mathematical Theory of the Navier-Stokes Equations. Vol. II. Nonlinear Steady Problems, Springer Tracts in Natural Philosophy 39, Springer, New York, 1994.

[L] O. A. Ladyzhenskaya, Mathematical Theory of Viscous Incompressible Flow, Nauka, Moscow, 1970 (in Russian).

[LSU] O. A. Ladyzhenskaya, V. A. Solonnikov and N. N. Ural'tseva, Linear and Quasilinear Equations of Parabolic Type, Nauka, Moscow, 1967 (in Russian).

[RZ] J. Rencławowicz and W. M. Zajączkowski, Existence of solutions to the Poisson equation in $L_{p}$-weighted spaces, Appl. Math. (Warsaw) 37 (2010), 1-12.

[S] V. A. Solonnikov, On general boundary value problems for Douglis-Nirenberg elliptic systems, Trudy Mat. Inst. Steklov. 92 (1966), 233-297 (in Russian).

[ZS] W. M. Zajączkowski and V. A. Solonnikov, On the Neumann problem for secondorder elliptic equations in a domain with edges at the boundary, Zap. Nauchn. Sem. LOMI 127 (1983), 7-48 (in Russian).

[Z1] W. M. Zajączkowski, Existence and regularity of solutions of some elliptic system in domains with edges, Dissertationes Math. 274 (1988), 95 pp.

[Z2] - Global regular nonstationary flow for the Navier-Stokes equations in a cylindrical pipe, Topol. Methods Nonlinear Anal. 26 (2005), 221-286. 
Joanna Rencławowicz

Institute of Mathematics

Polish Academy of Sciences

Śniadeckich 8

00-956 Warszawa, Poland

E-mail: jr@impan.gov.pl

\author{
Wojciech M. Zajączkowski \\ Institute of Mathematics \\ Polish Academy of Sciences \\ Sniadeckich 8 \\ 00-956 Warszawa, Poland \\ E-mail: wz@impan.gov.pl \\ and \\ Institute of Mathematics and Cryptology \\ Military University of Technology \\ Kaliskiego 2 \\ 00-908 Warszawa, Poland
}

Received on 3.11.2008;

revised version on 8.1.2010

(1974) 
